\title{
The potential of Ion Mobility Mass Spectrometry for high-throughput and high- resolution lipidomics
}

Christine Hinz, ch768@cam.ac.uk; Department of Biochemistry and Cambridge Systems Biology Centre, University of Cambridge, UK.

Sonia Liggi, s1584@cam.ac.uk; Department of Biochemistry and Cambridge Systems Biology Centre, University of Cambridge, UK.

Julian L Griffin, ilg40@cam.ac.uk; Department of Biochemistry and Cambridge Systems Biology Centre, University of Cambridge, UK.

$\mathrm{CH}$ and SL contributed equally to this work

\begin{abstract}
Lipids are a large and highly diverse family of biomolecules, which play essential structural, storage and signalling roles in cells and tissues. Although traditional mass spectrometry (MS) approaches used in lipidomics are highly sensitive and selective, lipid analysis remains challenging due to the chemical diversity of lipid structures, multiple isobaric species and incomplete separation using many forms of chromatography. Ion mobility (IM) separates ions in the gas phase based on their physicochemical properties. Addition of IM to the traditional lipidomic workflow both enhances separation of complex lipid mixtures, beneficial for lipid identification, and improves isomer resolution. Herein, we discuss the recent developments in IM-MS for lipidomics.
\end{abstract}

\section{Graphical Abstract}




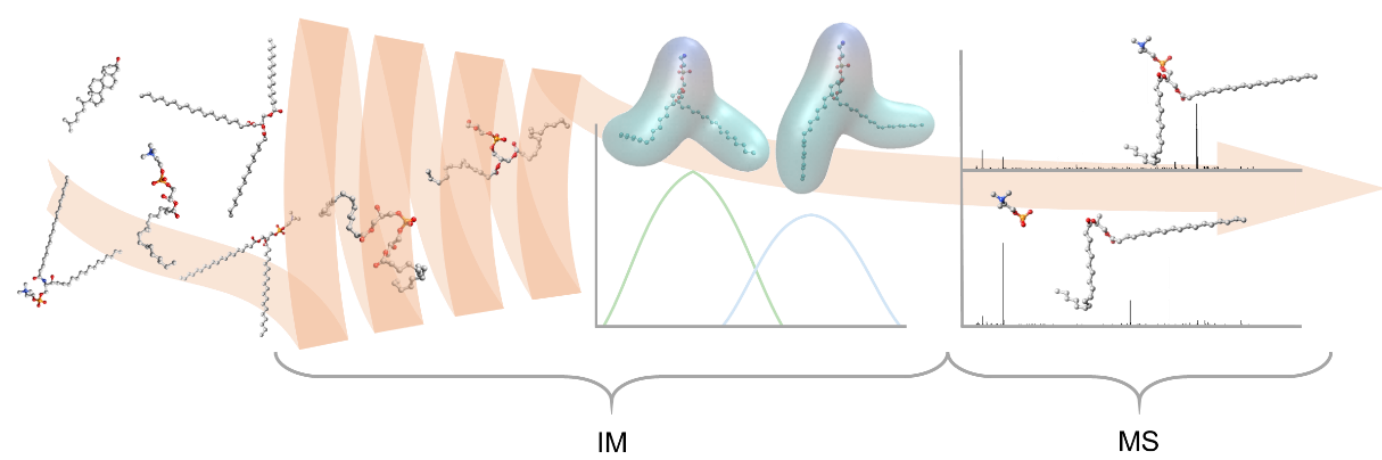

\section{Highlights}

- We review the current advantages of IM-MS spectrometry in lipidomics.

- Collision Cross-Section (CCS) values enhance confidence in lipid identification.

- IM separation coupled with fragmentation of precursor ions results in cleaner product ion spectra, allowing improved data interpretation.

- IM-MS improves separation of isomeric lipids.

\section{Abbreviations/Glossary}

$\begin{array}{ll}\text { BMP } & \text { bis(monoacylglycerol) phosphate } \\ \text { CCS } & \text { Collision Cross-Section } \\ \text { CE } & \text { cholesteryl ester } \\ \text { DAG } & \text { diacylglycerides } \\ \text { FA } & \text { fatty acid } \\ \text { GL } & \text { glycerolipid } \\ \text { GP } & \text { glycerophospholipid } \\ \text { IM } & \text { ion mobility } \\ \text { LC } & \text { liquid chromatography } \\ \text { Lyso-PL } & \text { lyso-phospholipid } \\ \text { MS } & \text { mass spectrometry } \\ \text { PC } & \text { phosphatidylcholine } \\ \text { PE } & \text { phosphatidylethanolamine } \\ \text { PG } & \text { phosphatidylglycerol } \\ \text { PI } & \text { phosphatidylinositol } \\ \text { PS } & \text { phosphatidylserine }\end{array}$




\section{Introduction}

Lipids are a highly diverse group of biomolecules comprising several complex lipid categories (detailed in Figure 1) which form an enormous network of structural, storage and signalling molecules within living organisms. Online databases such as LipidBlast [1], and LIPID MAPS [2] attempt to cover this large amount of information. However, the actual number of structurally distinct lipids is unknown. Today, the largest database is the LIPID MAPS Structure Database, which contains nearly 41,000 unique biologically relevant lipid structures, out of which approximately half is manually curated and half computationally generated (http://www.lipidmaps.org/data/structure/index.html, last accessed 25/07/2017).

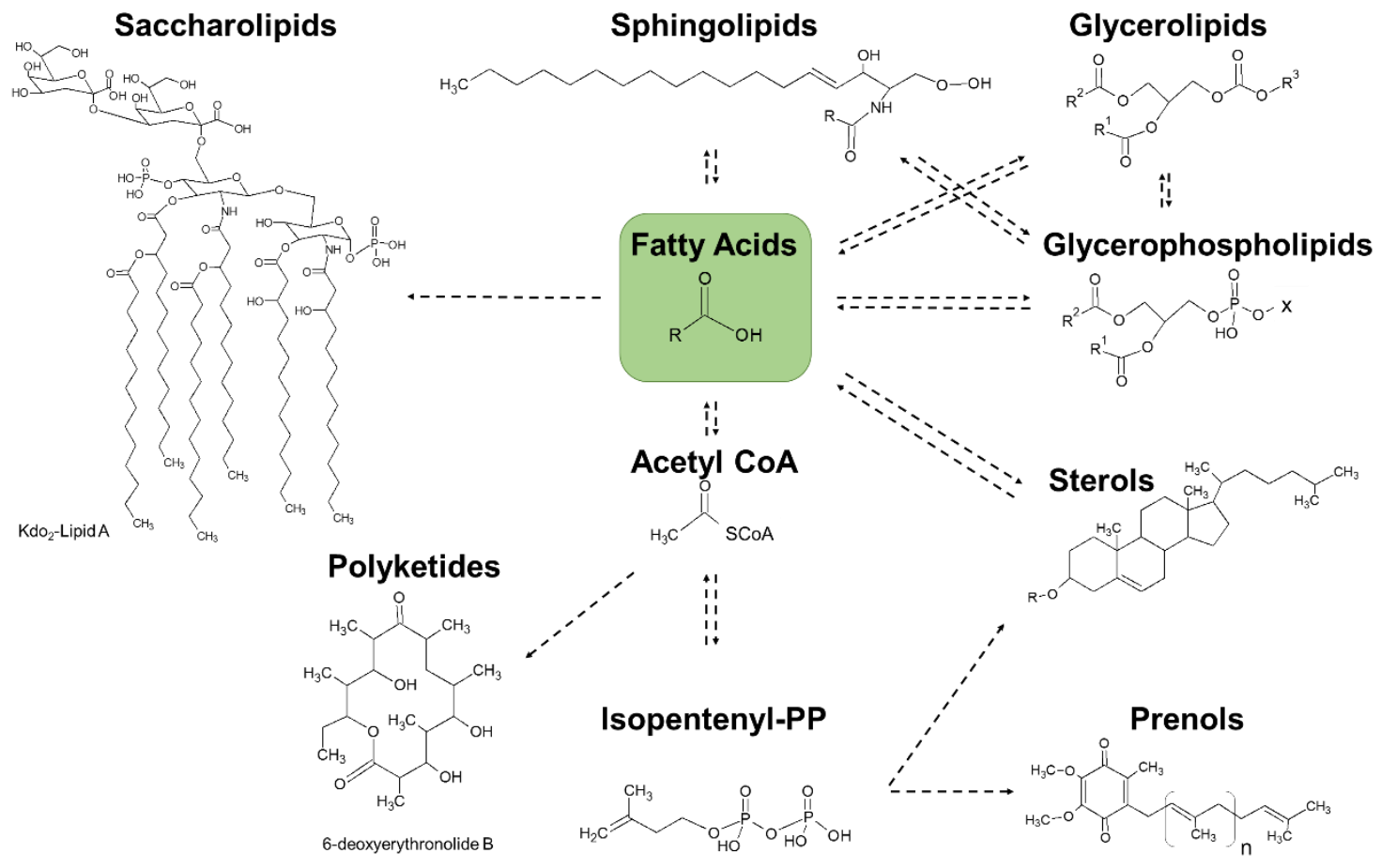

Figure 1: Fatty acid derived lipid categories. Fatty acids (FAs), which contain 2-40 carbon atoms and different degrees of unsaturation, are the smallest building blocks for much more complex lipid categories. These categories include sphingolipids, glycerolipids (GLs), glycerophospholipids (GPs), sterols, prenols, saccharolipids, and polyketides. Each category can be further divided into classes such as GPs into phosphatidylglycerols (PGs), phosphatidylethanolamines (PEs), phosphatidylcholines (PCs), phosphatidylserines (PSs), and phosphatidylinositols (PIs) depending on the head group (indicated with X).

The vast chemical space covered by the various lipid categories, along with the structural similarities within the same class, represents a significant analytical challenge in lipidomic studies, aiming to profile the entire lipid content (lipidome) of the studied samples. Currently, 
high-resolution mass spectrometry (MS) approaches either alone (shotgun MS) or coupled with liquid chromatography (LC/MS) are commonly used. However, insufficient separation of isomeric and isobaric species, very common in complex biological samples, is a major challenge. Therefore, further analytical separation methods are required to resolve isobaric lipid species, uncover the isomeric distribution of lipids, and to improve the confidence in lipid identification in biological samples.

Ion mobility (IM) is a gas-phase technique allowing the separation of ions based on their mobility through an inert gas (typically helium or nitrogen) under the influence of an electric field [3-6]. A number of different technologies in which IM is coupled with MS (IM-MS) have been developed, including the commercially available (1) Drift Time lon Mobility Spectrometry (DTIMS), developed by Agilent Technologies [7], (2) Field Asymmetric Ion Mobility Spectrometry/Differential Mobility Spectrometry (FAIMS/DMS) approach provided by $A B$ Sciex [8,9], (3) Travelling Wave Ion Mobility Spectrometry (TWIMS) developed by Waters [8,10], and (4) Trapped Ion Mobility Spectrometry (TIMS) developed by Bruker [11-13] . A detailed description of the theoretical concepts behind ion separation in these different technologies is not within the scope and space of this brief review, and hence we refer the reader to recent reviews in the field [4-6]. IM-MS has been shown to provide significant analytical improvements for a variety of chemical classes. Compared to MS only, using IMMS as additional separation reduces interfering signals in the gas phase, thus increasing spectral clarity, enhancing peak capacity, and improving both selectivity and sensitivity [1419]. In addition, the Collision Cross-Section (CCS), a physicochemical descriptor correlated to shape, size and charge of ions, can be calculated with most commercially available instruments by considering the experimental conditions and the observed mobility of ions. Both CCS values and accurate mass are highly reproducible $(<2 \%,<5 \mathrm{ppm}$, respectively) $[17,19,20]$ and can be used to discriminate closely related species by both size and mass. In addition, coupling LC to IM-MS [6] allows compound characterisation in multiple dimensions, making LC/IM-MS a promising approach for the comprehensive analysis of complex lipid mixtures $[17,19]$.

Due to the emerging developments in both hardware and software, IM-MS has recently been implemented in a variety of lipidomic workflows, which are reviewed in the following sections.

\section{Improved lipid identification by IM-MS}

Currently, compounds discovered in a lipidomic workflow are putatively identified based on accurate mass match with online databases such as LIPID MAPS or LipidBlast. However, as accurate mass only provides the molecular formula, it could correspond to a number of species belonging to different lipid classes. Therefore, further information about the compound's 
physicochemical characteristics are required to allow a more accurate identification. It has been shown that the CCS values of FAs and PCs are highly correlated with both the lipid chain length and the degree of unsaturation [21-23] (Figure 2 A and B). In particular, larger CCS values are found for saturated species, whose acyl chains extend under the electric field, while unsaturated bonds confer a bent structure in the acyl chain resulting in smaller CCS values $[21,22]$. The CCS is influenced not only by the degree of saturation, but more generally by the structural characteristics of compounds. Thus, different lipid categories, such as GPs and sphingolipids, have distinct mobility behaviours by which they can be separated. This allows a more selective analysis of lipids belonging to different categories but sharing an identical accurate mass [24]. In addition, the distinct mobility trends observed not only between lipid categories but also among different biomolecular families (such as lipids, peptides and oligonucleotides) improve data interpretation in imaging MS [19,25-32]. In this technique, lipids are analysed directly from tissue slices without additional extraction and/or purification steps, allowing simultaneous CCS evaluation and lipid localisation in tissue [33]. Similarly, different lipid classes belonging to the same category can be separated by mobility based on their head groups (shown for PEs and PC in Figure $2 \mathbf{C}$ ); however, separation is less efficient than between lipid categories, with some overlapping CCS values between certain species $[19,26]$. Overall, these lipid category/class specific mobility behaviours decrease the false discovery rate providing a higher degree of confidence in lipid identification [17,22,24,26,34]. 
A

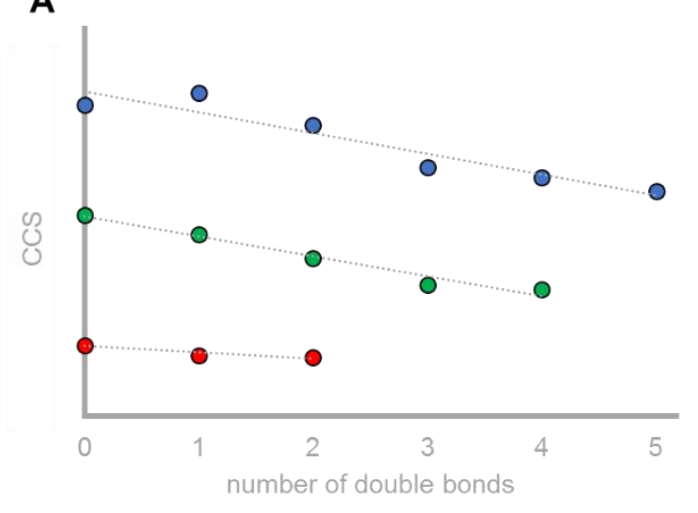

B

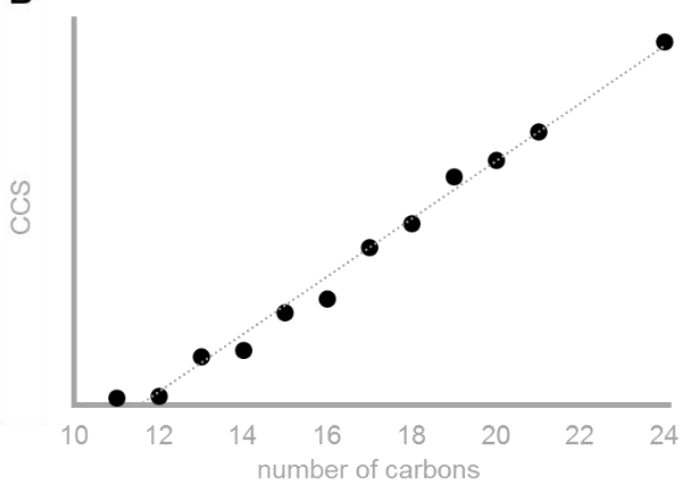

C
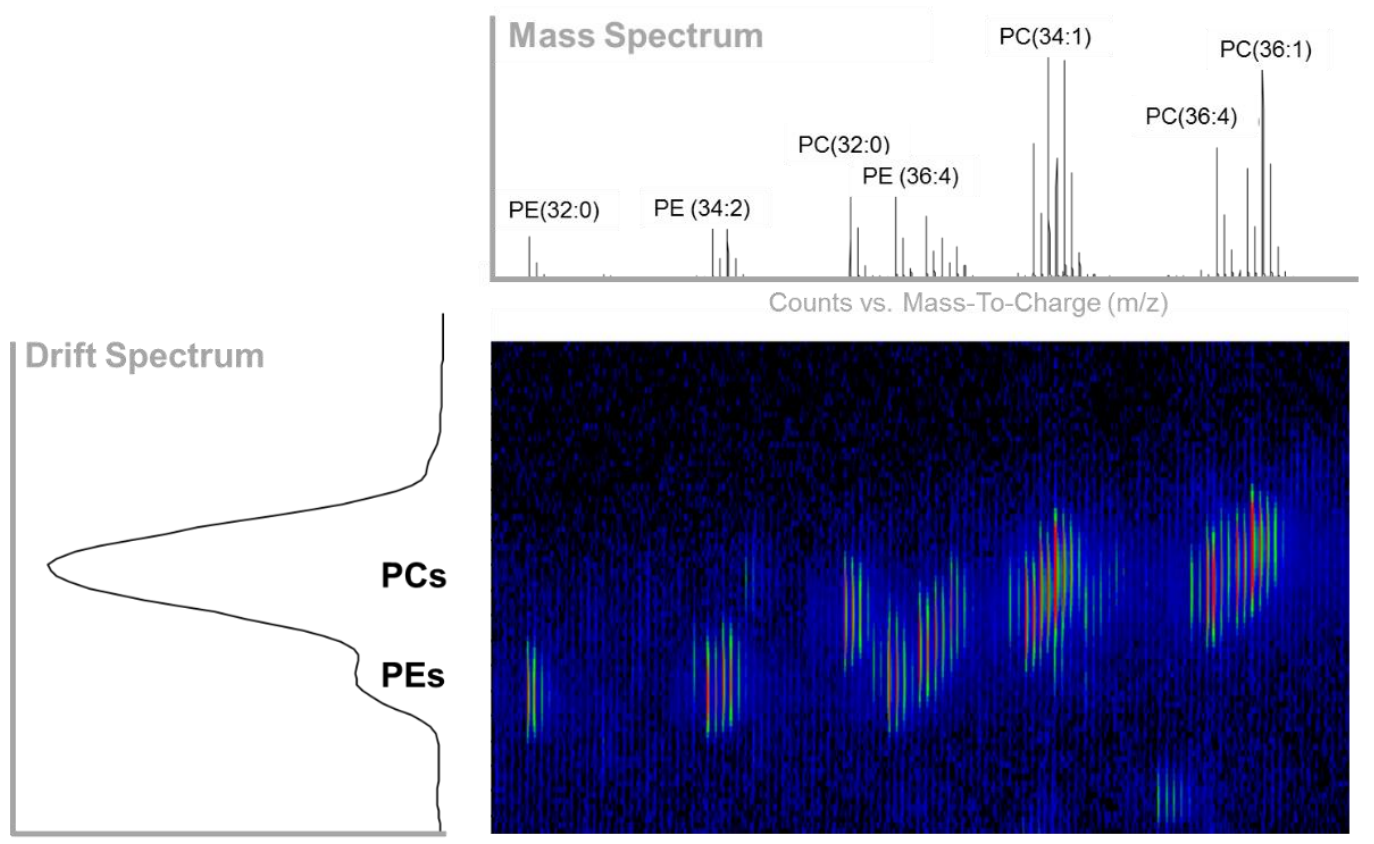

Counts vs. Drift Time (ms)

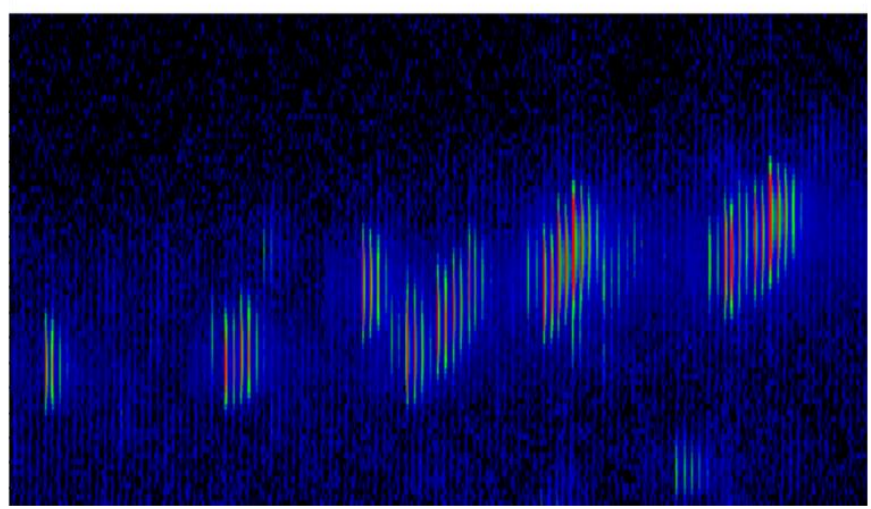

Drift Time (ms) vs. m/z

Figure 2. Improved identification of lipids by IM-MS. The CCS values of fatty acids increase with both (A) number of double bonds (C16-C20 indicates the number of carbon atoms and $x$ the number of double bonds) and

(B) lipid chain length. (C) PES and PCs are mobility separated based on their head group using a DTIMS-MS instrument.

In order to validate experimentally obtained CCS values, several research groups have developed their own CCS databases by analysing synthetic lipid standards [17,19,35]. However, due to the limited number of commercially available standards, in silico CCS determination may represent a promising approach to cover the lipidome of complex biological matrices, as demonstrated for lipids and other biomolecules [36-40].

Traditionally, lipids are identified using either targeted or untargeted fragmentation of precursor ions to obtain diagnostic product ions in high-resolution spectra [41-46]. The addition of IM to targeted workflows, in which precursor ion masses are selected for fragmentation, has been shown to reduce background noise, simplifying interpretation of the spectra and improving the overall detection limits in lipid mixtures $[14,16,30]$. This is very important when analysing low abundant but biologically relevant species. On the other hand, 
targeted methods may struggle with poor duty cycles and decreased sensitivity when too many ion masses are fragmented. Untargeted fragmentation can overcome these sensitivity issues as no precursor ion masses are selected. However, data interpretation is challenging due to presence of multiple product ion masses deriving from co-eluting species in the same spectrum. This is especially true for lipids, as their fragments share a high degree of similarity [47]. It has been demonstrated that addition of IM to untargeted lipidomics allows mobilityalignment of fragment ions to their precursors when fragmentation takes place following mobility separation [48-51] (Figure 3).In contrast, fragmentation prior to IM separation allows CCS evaluation of diagnostic product ions as an additional identifying factor [52-54]. Therefore, both approaches improve data interpretation and enhance lipid identification in untargeted workflows. 
A Chromatographic separation

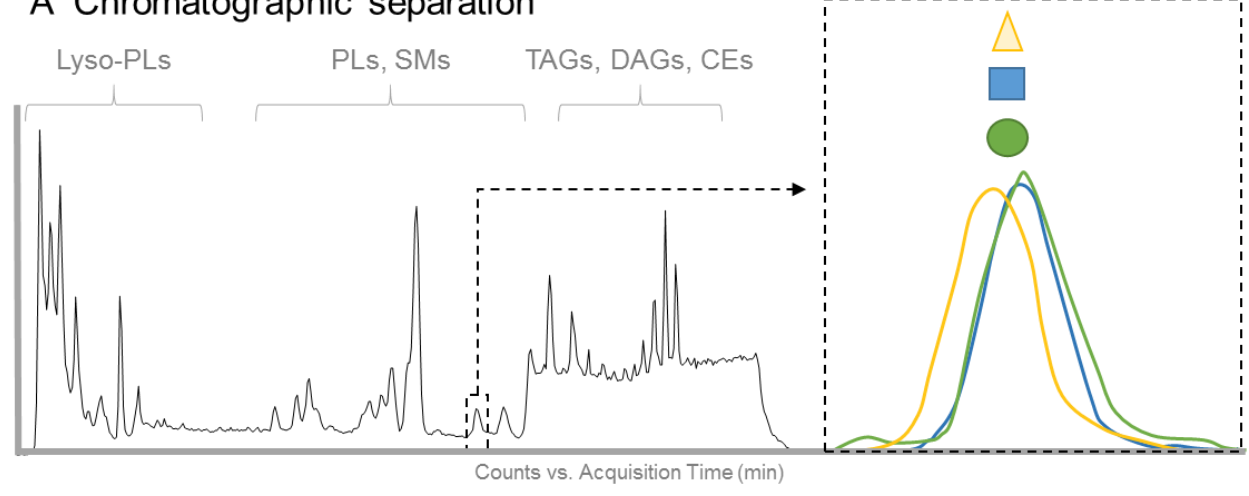

B Mobilogram

C Precursor ion spectrum
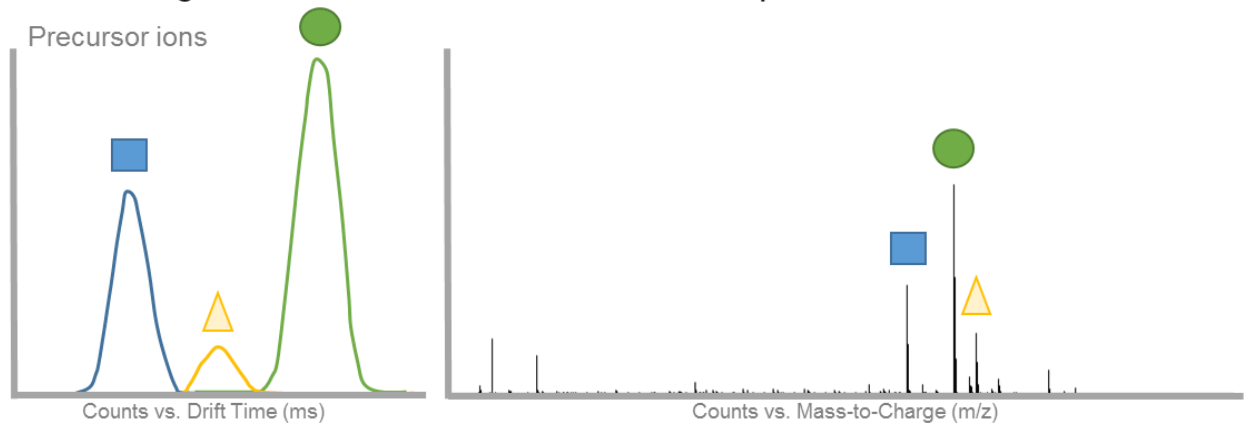

B Mobilogram

E Product ion spectrum

Precursor and

Product ions
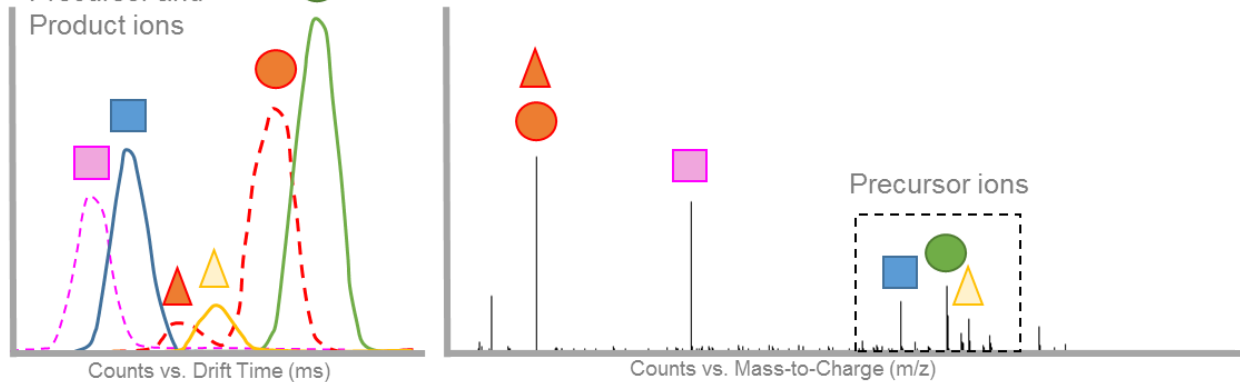

F Improved interpretation of product ion spectra

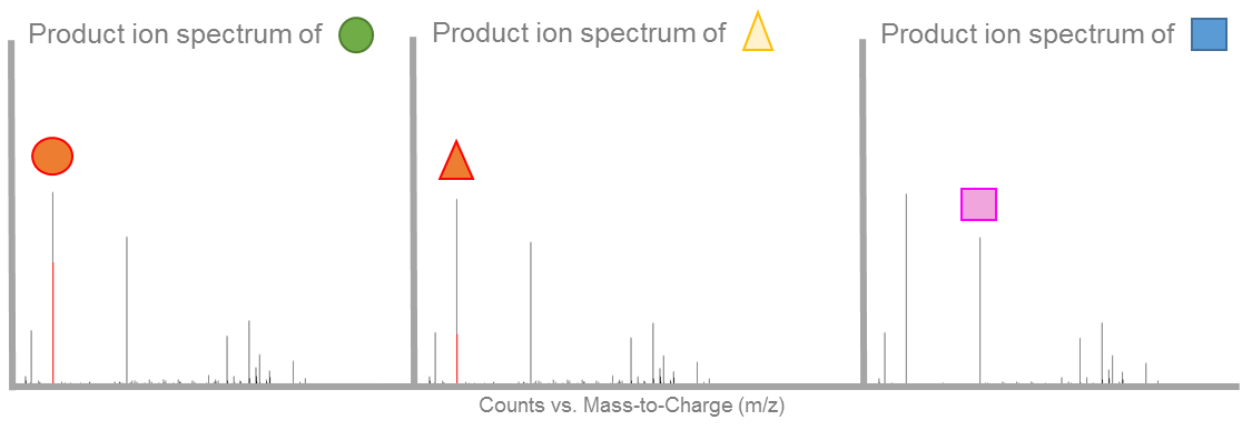

Figure 3: Ion mobility separation of co-eluting lipids and their product ions. (A) Chromatographic separation of a complex mixture of lipids such as tissue extracts containing lyso-phospholipids (lyso-PLs), glycerophospholipids (GPs), sphingomyelins (SMs), triacylglycerides (TAGs), diacylglycerides (DAGs) and cholesteryl-esters (CEs) with the extracted ion chromatogram of insufficiently separated lipids species magnified in yellow, blue, and green. These species can be separated by (B) ion mobility and (C) accurate mass. For lipid identification collision-induced fragmentation is applied. However, untargeted fragmentation of all precursor ions present in a sample results in a mix of both precursor (solid) and product (dashed) ion (D) mobilities in the mobilogram and $(E)$ ion masses in the product ion spectrum. As IM separation occurs before fragmentation, product ions can be mobility-aligned to their precursor ions consequently improving interpretation of product ion spectra $(F)$. In addition, identical product ions deriving from different precursor ions (such as red deriving from green and yellow) can be assigned to their precursors in correct proportions (marked in red). 


\section{Isomer separation}

One of the major challenges in lipid analysis is isomer separation, with regio- (such as sn1 or sn2 for GPs), positional (position of double bond), or geometric (cis/trans conformation of the double bond, also known as Z/E) isomers commonly found in complex lipid mixtures (
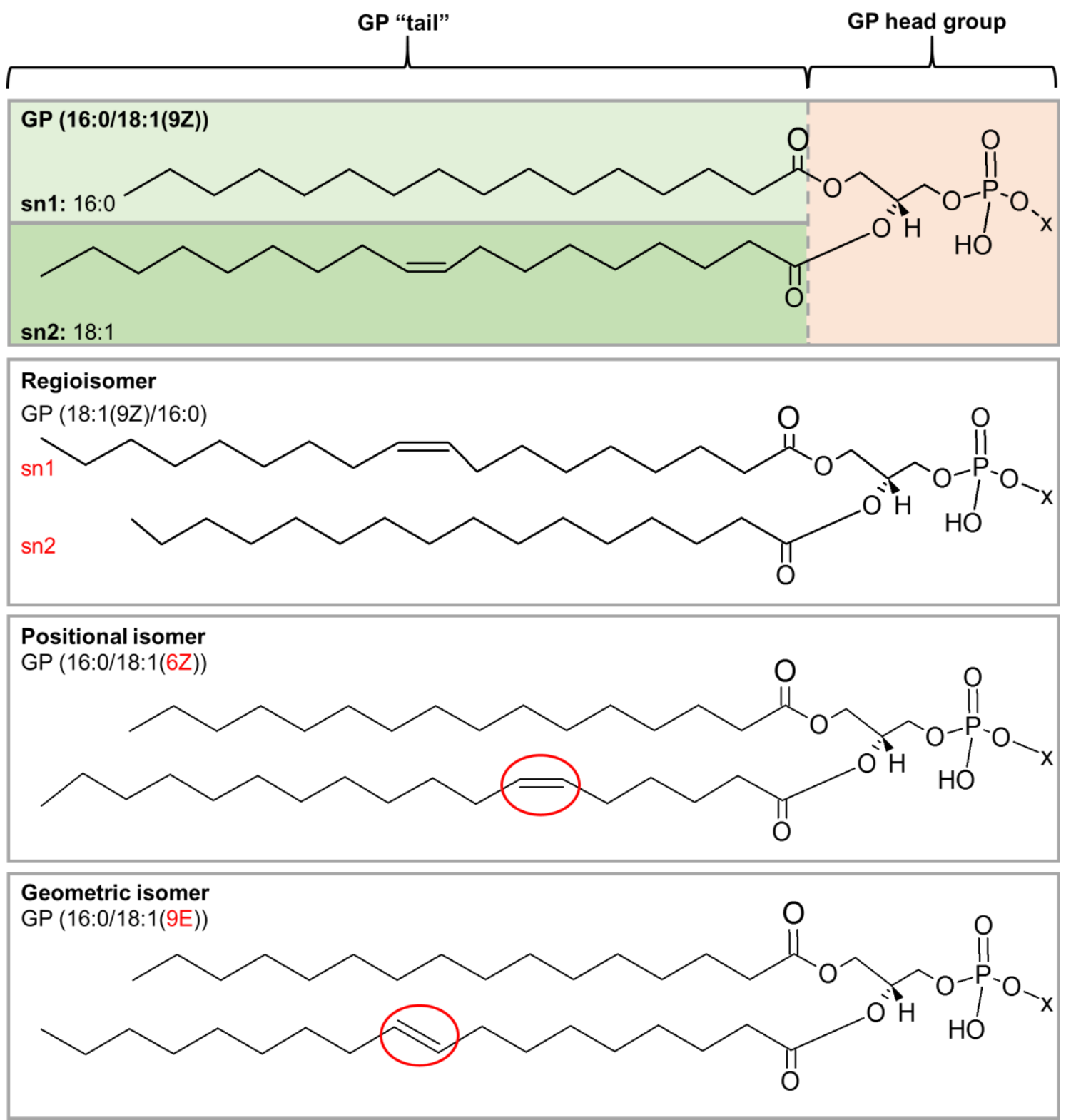

Figure 4). Although LC can separate some isomeric compounds, several will co-elute and exhibit identical fragmentation spectra, making their identification difficult. IM can improve separation of isobaric and isomeric lipids due to differences in their structure and shape [24,55-57]. However, all three dimensions of LC, IM and MS are needed to target isomer 
separation which still may not be complete $[22,49,58]$, although it can be enhanced by the use of different inert gases in the mobility chamber [24,55].

The conformational differences between geometrical isomers, and in particular the curled structure caused by a cis- double bond in contrast to a more open conformation conferred by trans geometry, allows separation between cis and trans isomers of GPs [22]. In addition, cisunsaturated positional isomers of FAs could be resolved, while their equivalents containing trans-double bonds could not be distinguished due to smaller differences in shape caused by the trans geometry compared to cis [22]. Comparably, a double bond closer to the head group of GPs causes a larger structure than when positioned further down the acyl chain, allowing separation of PC positional isomers [59]. In contrast, positional isomers of mono-unsaturated FAs could only be separated when injected individually, while their mixtures could not be resolved due to only minor differences among the isomers conformations [23]. Separation of GP regioisomers was observed in targeted [48], multidimensional (IMS ${ }^{3}-\mathrm{MS}$ ) [34] approaches, with a more elongated three-dimensional conformation observed when the longer FA chain is linked to the sn1 position [22]. Interestingly, the opposite holds for lysoPC (which only have a single acyl chain attached to either sn1 or sn2 position of the GP backbone), where a FA in sn2 position results in a larger cross-section for the ion [22].

The separation of isomers whose spatial conformations are too similar can be enhanced with several strategies, such as using instruments with a higher IM resolving power [50,55,60]. Indeed, on a lower resolution instrument the two structural isomers PG and bis(monoacylglycerol) phosphate (BMP) could be distinguished only following evaluation of their fragmentation pattern [61], while their separation as native ions was possible by increasing the IM resolving power [22]. However, the choice of commercially available IM-MS instruments offering a high resolving power today is limited [50]. To overcome this deficiency, derivatization can be used to increase the shape differences between isomers and was shown to successfully resolve synthetically generated steroid isoforms [62]. Similarly, the formation of adducts with alkali ions can enhance isomers separation thanks to the differences in coordination geometries, as demonstrated for steroid epimers, ceramide stereoisomers, and diastereoisomeric forms of sphingosine [22,59,63]. Furthermore, interaction of alkali ions with the double bond, in contrast to protonation which happens in the head group, can increase the separation between sodiated and potassiated adducts of PC geometrical and regioisomers [64]. Likewise, the ability of silver to bind both lone pairs of electrons from heteroatoms and $\pi$ electrons of the double bond allowed resolution of PC as well as $\mathrm{GL}$ isomers [65,66]. Another approach to assign double bond positions in complex lipids and distinguish GL isomers is ozone-induced dissociation (OzID) [67]. Although not the main focus of their study, Poad and co-workers observed mobility separation between precursor and OzID product ions, indicating 
OzID coupled with IM as a promising approach to gain further understanding into lipid structures.
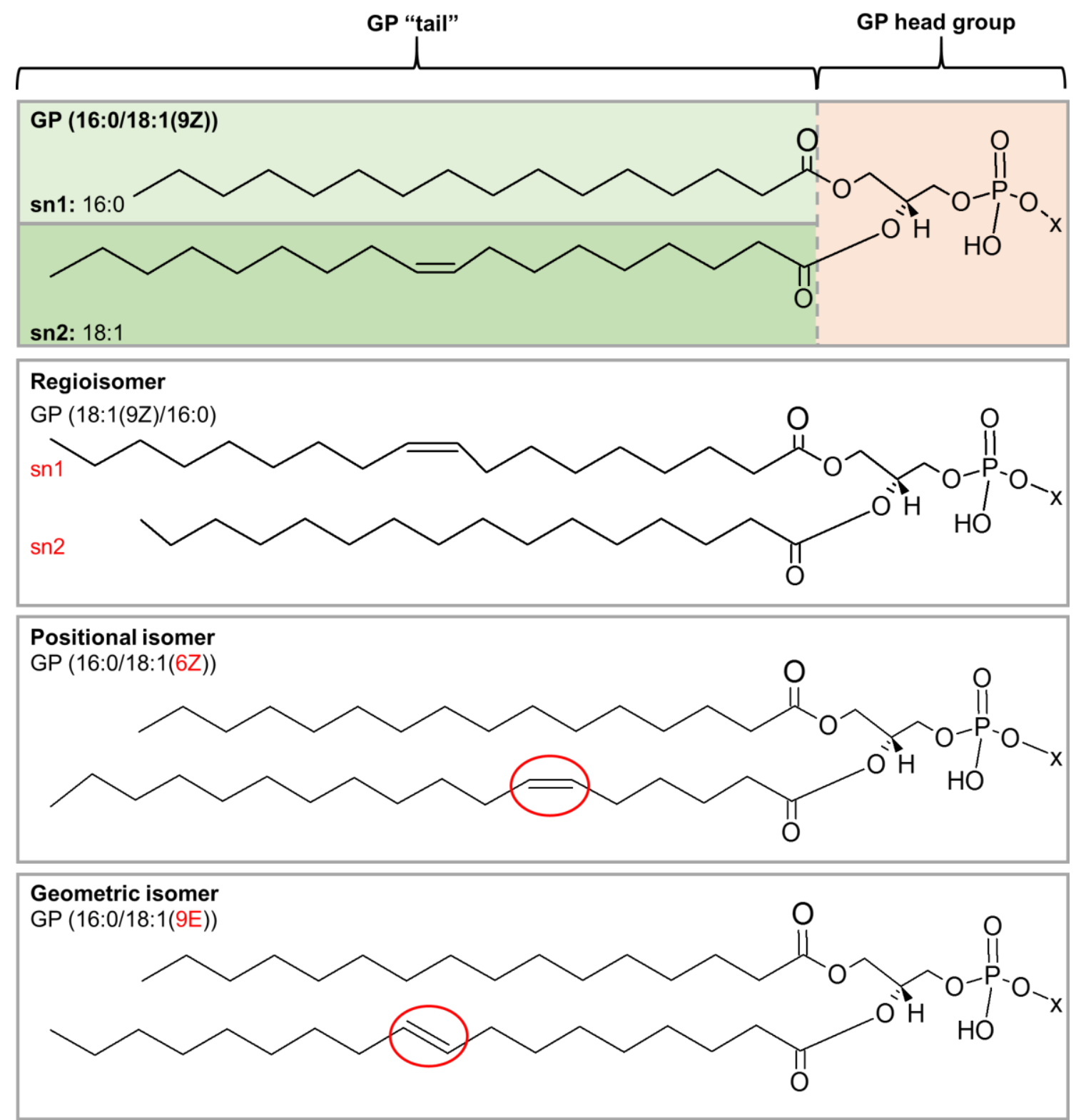

Figure 4: Isomeric structures of glycerophospholipids. Glycerophospholipids (GPS) consist of a polar head group (glycerol, choline, ethanolamine, inositol, or serine indicated as " $x$ ") and two fatty acyl groups at sn1 (16:0) and sn2 (18:1 (9Z)) position forming the GP tail. Three types of isomers are possible: a regioisomer presenting the fatty acyl chains in inverted positions; a positional isomer, containing the double bond in different position (C6 instead of C9), and a geometric isomer, whose double bond is in trans (E) conformation.

\section{Conclusion}


The chemical complexity of lipids makes highly sensitive and selective analytical tools crucial for their analysis in complex mixtures such as extracts of biological tissues. Recent technical developments in both hardware and software enabled researchers to implement IM-MS into their analytical workflows with four major improvements in lipidomic analysis. First, IM-MS improves separation of lipids in complex mixtures, crucial to resolving isobaric species in biological samples. Second, IM improves the spectral clarity of product ion spectra derived from fragmentation experiments. This is important in both lipid identification and structural elucidation, which are challenging tasks due to isomeric nature of many lipid species. On the other hand, analysis of product ion spectra derived from untargeted fragmentation acquisitions remains challenging due to the required powerful processing tools. Third, IM improves separation of isomeric lipids. However, to completely uncover the isomeric distribution in complex lipid mixtures, a higher resolving power than is commercially available today may be required. Last, CCS values obtained from IM-MS analysis effectively increase confidence in lipid identification. However, validation of CCS values is restricted to the limited number of commercially available lipid standards. Therefore, in silico CCS prediction is an important tool to improve identification efficiency in lipidomics. Concluding, IM-MS is a promising analytical tool for lipidomic studies that has the potential of providing new insights into lipid biology.

\section{Acknowledgements}

This work was supported by Agilent Technologies, Santa Clara, USA; and the Medical Research Council, UK (MC UP A90 1006 \& MC PC 13030).

\section{Bibliography}

1. Kind T, Liu K-H, Lee DY, DeFelice B, Meissen JK, Fiehn O: LipidBlast in silico tandem mass spectrometry database for lipid identification. Nat. Methods 2013, 10:755758.

2. Sud M, Fahy E, Cotter D, Brown A, Dennis EA, Glass CK, Merrill AH, Murphy RC, Raetz CRH, Russell DW, et al.: LMSD: LIPID MAPS structure database. Nucleic Acids Res. 2007, 35:D527-D532.

3. Kanu AB, Dwivedi P, Tam M, Matz L, Hill HH: lon mobility-mass spectrometry. J. Mass Spectrom. 2008, 43:1-22.

4. Lanucara F, Holman SW, Gray CJ, Eyers CE: The power of ion mobility-mass 
spectrometry for structural characterization and the study of conformational dynamics. Nat. Chem. 2014, 6:281-294.

5. Lapthorn C, Pullen F, Chowdhry BZ: Ion mobility spectrometry-mass spectrometry (IMS-MS) of small molecules: Separating and assigning structures to ions. Mass Spectrom. Rev. 2013, 32:43-71.

-6. May JC, McLean JA: Ion Mobility-Mass Spectrometry: Time-Dispersive Instrumentation. Anal. Chem. 2015, 87:1422-1436.

Detailed description of the available IM technologies.

7. Ibrahim YM, Baker ES, Danielson WF, Norheim R V., Prior DC, Anderson GA, Belov ME, Smith RD: Development of a new ion mobility time-of-flight mass spectrometer. Int. J. Mass Spectrom. 2015, 377:655-662.

8. Shvartsburg AA, Smith RD: Fundamentals of Traveling Wave Ion Mobility Spectrometry. Anal. Chem. 2008, 80:9689-9699.

9. Schneider BB, Nazarov EG, Londry F, Vouros P, Covey TR: Differential mobility spectrometry/mass spectrometry history, theory, design optimization, simulations, and applications. Mass Spectrom. Rev. 2016, 35:687-737.

10. Giles K: Travelling wave ion mobility. Int. J. Ion Mobil. Spectrom. 2013, 16:69-69.

11. Michelmann K, Silveira JA, Ridgeway ME, Park MA: Fundamentals of Trapped lon Mobility Spectrometry. J. Am. Soc. Mass Spectrom. 2015, 26:14-24.

12. Hernandez DR, DeBord JD, Ridgeway ME, Kaplan DA, Park MA, Fernandez-Lima F: Ion dynamics in a trapped ion mobility spectrometer. Analyst 2014, 139:1913-1921.

13. Fernandez-Lima F, Kaplan DA, Suetering J, Park MA: Gas-phase separation using a trapped ion mobility spectrometer. Int. J. Ion Mobil. Spectrom. 2011, 14:93-98.

-14. Baker PRS, Armando AM, Campbell JL, Quehenberger O, Dennis EA: Threedimensional enhanced lipidomics analysis combining UPLC, differential ion mobility spectrometry, and mass spectrometric separation strategies. J. Lipid Res. 2014, 55:2432-2442.

Differential ion mobility spectrometry coupled with LC/MS was used to separate phospholipid classes by their head group reducing background interference and increasing resolution of lipids compared with LC alone.

15. Counterman AE, Hilderbrand AE, Barnes CAS, Clemmer DE: Formation of peptide 
aggregates during ESI: Size, charge, composition, and contributions to noise. J. Am. Soc. Mass Spectrom. 2001, 12:1020-1035.

16. Lintonen TPI, Baker PRS, Suoniemi M, Ubhi BK, Koistinen KM, Duchoslav E, Campbell JL, Ekroos K: Differential Mobility Spectrometry-Driven Shotgun Lipidomics. Anal. Chem. 2014, 86:9662-9669.

-17. May JC, Goodwin CR, Lareau NM, Leaptrot KL, Morris CB, Kurulugama RT, Mordehai A, Klein C, Barry W, Darland E, et al.: Conformational Ordering of Biomolecules in the Gas Phase: Nitrogen Collision Cross Sections Measured on a Prototype High Resolution Drift Tube Ion Mobility-Mass Spectrometer. Anal. Chem. 2014, 86:2107-2116.

First reported database of empirically derived nitrogen CCS measurements from drift tube ion mobility for four molecular classes (salts, lipids, peptides, and carbohydrates; 594 compounds in total).

18. Pacini T, Fu W, Gudmundsson S, Chiaravalle AE, Brynjolfson S, Palsson BO, Astarita G, Paglia G: Multidimensional Analytical Approach Based on UHPLC-UV-Ion MobilityMS for the Screening of Natural Pigments. Anal. Chem. 2015, 87:2593-2599.

••19. Paglia G, Angel P, Williams JP, Richardson K, Olivos HJ, Thompson JW, Menikarachchi L, Lai S, Walsh C, Moseley A, et al.: Ion Mobility-Derived Collision Cross Section As an Additional Measure for Lipid Fingerprinting and Identification. Anal. Chem. 2015, 87:1137-1144.

More than 200 lipids were analysed using traveling wave IM-MS. The obtained CCS values, which are reported, increased confidence in lipid identification.

20. Paglia G, Williams JP, Menikarachchi L, Thompson JW, Tyldesley-Worster R, Halldórsson S, Rolfsson O, Moseley A, Grant D, Langridge J, et al.: Ion Mobility Derived Collision Cross Sections to Support Metabolomics Applications. Anal. Chem. 2014, 86:3985-3993.

21. Kim HI, Kim H, Pang ES, Ryu EK, Beegle LW, Loo JA, Goddard WA, Kanik I: Structural Characterization of Unsaturated Phosphatidylcholines Using Traveling Wave Ion Mobility Spectrometry. Anal. Chem. 2009, 81:8289-8297.

22. Kyle JE, Zhang X, Weitz KK, Monroe ME, Ibrahim YM, Moore RJ, Cha J, Sun X, Lovelace ES, Wagoner $\mathrm{J}$, et al.: Uncovering biologically significant lipid isomers with liquid chromatography, ion mobility spectrometry and mass spectrometry. Analyst 2016, 141:1649-1659. 
23. Zhang F, Guo S, Zhang M, Zhang Z, Guo Y: Characterizing ion mobility and collision cross section of fatty acids using electrospray ion mobility mass spectrometry. J. Mass Spectrom. 2015, 50:906-913.

24. Shvartsburg AA, Isaac G, Leveque N, Smith RD, Metz TO: Separation and Classification of Lipids Using Differential Ion Mobility Spectrometry. J. Am. Soc. Mass Spectrom. 2011, 22:1146-1155.

25. Jackson SN, Ugarov M, Egan T, Post JD, Langlais D, Albert Schultz J, Woods AS: MALDI-ion mobility-TOFMS imaging of lipids in rat brain tissue. J. Mass Spectrom. 2007, 42:1093-1098.

26. Jackson SN, Ugarov M, Post JD, Egan T, Langlais D, Schultz JA, Woods AS: A study of phospholipids by ion mobility TOFMS. J. Am. Soc. Mass Spectrom. 2008, 19:16551662.

27. Jackson SN, Barbacci D, Egan T, Lewis EK, Schultz JA, Woods AS: MALDI-ion mobility mass spectrometry of lipids in negative ion mode. Anal. Methods 2014, 6:50015007.

28. Kliman M, Vijayakrishnan N, Wang L, Tapp JT, Broadie K, McLean JA: Structural mass spectrometry analysis of lipid changes in a Drosophila epilepsy model brain. Mol. Biosyst. 2010, 6:958.

29. McLean JA, Ridenour WB, Caprioli RM: Profiling and imaging of tissues by imaging ion mobility-mass spectrometry. J. Mass Spectrom. 2007, 42:1099-1105.

30. Paglia G, Kliman M, Claude E, Geromanos S, Astarita G: Applications of ionmobility mass spectrometry for lipid analysis. Anal. Bioanal. Chem. 2015, 407:4995-5007.

31. Woods AS, Ugarov M, Egan T, Koomen J, Gillig KJ, Fuhrer K, Gonin M, Schultz JA: Lipid/Peptide/Nucleotide Separation with MALDI-Ion Mobility-TOF MS. Anal. Chem. 2004, 76:2187-2195.

32. Woods AS, Jackson SN: The Application and Potential of Ion Mobility Mass Spectrometry in Imaging MS with a Focus on Lipids. In Methods Mol Biol. Edited by Rubakhin SS, Sweedler J V. Humana Press; 2010:99-111.

33. Ridenour WB, Kliman M, McLean JA, Caprioli RM: Structural Characterization of Phospholipids and Peptides Directly from Tissue Sections by MALDI Traveling-Wave Ion Mobility-Mass Spectrometry. Anal. Chem. 2010, 82:1881-1889.

34. Trimpin S, Tan B, Bohrer BC, O'Dell DK, Merenbloom SI, Pazos MX, Clemmer DE, 
Walker JM: Profiling of phospholipids and related lipid structures using multidimensional ion mobility spectrometry-mass spectrometry. Int. J. Mass Spectrom. 2009, 287:58-69.

35. Hines KM, Herron J, Xu L: Assessment of altered lipid homeostasis by HILIC-ion mobility-mass spectrometry-based lipidomics. J. Lipid Res. 2017, 58:809-819.

36. Shah AR, Agarwal K, Baker ES, Singhal M, Mayampurath AM, Ibrahim YM, Kangas LJ, Monroe ME, Zhao R, Belov ME, et al.: Machine learning based prediction for peptide drift times in ion mobility spectrometry. Bioinformatics 2010, 26:1601-1607.

37. Soper-Hopper MT, Petrov AS, Howard JN, Yu SS, Forsythe JG, Grover MA, Fernandez FM: Collision cross section predictions using 2-dimensional molecular descriptors. Chem Comm 2017, 53:7624-7627.

38. Zhou Z, Shen X, Tu J, Zhu Z-J: Large-Scale Prediction of Collision Cross-Section Values for Metabolites in Ion Mobility-Mass Spectrometry. Anal. Chem. 2016, 88:1108411091.

39. Zhou Z, Xiong X, Zhu Z-J: MetCCS predictor: a web server for predicting collision cross-section values of metabolites in ion mobility-mass spectrometry based metabolomics. Bioinformatics 2017, 33:2235-2237.

•40. Zhou Z, Tu J, Xiong X, Shen X, Zhu Z-J: LipidCCS: Prediction of Collision CrossSection Values for Lipids with High Precision to Support Ion Mobility-Mass Spectrometry based Lipidomics. Anal. Chem. 2017, doi:10.1021/acs.analchem.7b02625.

Prediction of CCS values for lipids using a support vector regression (SVR) based model, with a median relative error of $\sim 1 \%$.

41. Ahmed Z, Mayr M, Zeeshan S, Dandekar T, Mueller MJ, Fekete A: Lipid-Pro: a computational lipid identification solution for untargeted lipidomics on dataindependent acquisition tandem mass spectrometry platforms. Bioinformatics 2015, 31:1150-1153.

42. Cajka T, Fiehn O: Comprehensive analysis of lipids in biological systems by liquid chromatography-mass spectrometry. TrAC Trends Anal. Chem. 2014, 61:192-206.

43. Castro-Perez JM, Kamphorst J, DeGroot J, Lafeber F, Goshawk J, Yu K, Shockcor JP, Vreeken RJ, Hankemeier T: Comprehensive LC-MSE Lipidomic Analysis using a Shotgun Approach and Its Application to Biomarker Detection and Identification in Osteoarthritis Patients. J. Proteome Res. 2010, 9:2377-2389. 
44. Gallart-Ayala H, Courant F, Severe S, Antignac J-P, Morio F, Abadie J, Le Bizec B: Versatile lipid profiling by liquid chromatography-high resolution mass spectrometry using all ion fragmentation and polarity switching. Preliminary application for serum samples phenotyping related to canine mammary cancer. Anal. Chim. Acta 2013, 796:7583.

45. Kyle JE, Crowell KL, Casey CP, Fujimoto GM, Kim S, Dautel SE, Smith RD, Payne SH, Metz TO: LIQUID: an-open source software for identifying lipids in LC-MS/MS-based lipidomics data. Bioinformatics 2017, 33:1744-1746.

46. Simons B, Kauhanen D, Sylvänne T, Tarasov K, Duchoslav E, Ekroos K: Shotgun Lipidomics by Sequential Precursor Ion Fragmentation on a Hybrid Quadrupole Timeof-Flight Mass Spectrometer. Metabolites 2012, 2:195-213.

47. Murphy RC: Tandem Mass Spectrometry of Lipids. Royal Society of Chemistry; 2014.

48. Castro-Perez J, Roddy TP, Nibbering NMM, Shah V, McLaren DG, Previs S, Attygalle $A B$, Herath K, Chen Z, Wang S-P, et al.: Localization of Fatty Acyl and Double Bond Positions in Phosphatidylcholines Using a Dual Stage CID Fragmentation Coupled with Ion Mobility Mass Spectrometry. J. Am. Soc. Mass Spectrom. 2011, 22:1552-1567.

49. Damen CWN, Isaac G, Langridge J, Hankemeier T, Vreeken RJ: Enhanced lipid isomer separation in human plasma using reversed-phase UPLC with ion-mobility/highresolution MS detection. J. Lipid Res. 2014, 55:1772-1783.

-50. Paglia G, Astarita G: Metabolomics and lipidomics using traveling-wave ion mobility mass spectrometry. Nat. Protoc. 2017, 12:797-813.

The authors presented an LC/IM-MS protocol for lipidomic analysis using a travelling wave IM instrument.

51. Shah V, Castro-Perez JM, McLaren DG, Herath KB, Previs SF, Roddy TP: Enhanced data-independent analysis of lipids using ion mobility-TOFMS $E$ to unravel quantitative and qualitative information in human plasma. Rapid Commun. Mass Spectrom. 2013, 27:2195-2200.

• •52. Zemski Berry KA, Barkley RM, Berry JJ, Hankin JA, Hoyes E, Brown JM, Murphy RC: Tandem Mass Spectrometry in Combination with Product Ion Mobility for the Identification of Phospholipids. Anal. Chem. 2017, 89:916-921.

Zemski Berry and co-workers used concerted tandem and travelling wave IM-MS to separate phospholipid product ions derived from collision-induced fragmentation. CCS values for 
diagnostic product ions were reported to be beneficial for structural identification of lipids in human serum.

53. Wickramasekara S, Zandkarimi F, Morré J, Kirkwood J, Legette L, Jiang Y, Gombart A, Stevens J, Maier C: Electrospray Quadrupole Travelling Wave Ion Mobility Time-ofFlight Mass Spectrometry for the Detection of Plasma Metabolome Changes Caused by Xanthohumol in Obese Zucker (fa/fa) Rats. Metabolites 2013, 3:701-717.

54. Knapman TW, Berryman JT, Campuzano I, Harris SA, Ashcroft AE: Considerations in experimental and theoretical collision cross-section measurements of small molecules using travelling wave ion mobility spectrometry-mass spectrometry. Int. J. Mass Spectrom. 2010, 298:17-23.

- •55. Bowman AP, Abzalimov RR, Shvartsburg AA: Broad Separation of Isomeric Lipids by High-Resolution Differential Ion Mobility Spectrometry with Tandem Mass Spectrometry. J. Am. Soc. Mass Spectrom. 2017, 28:1552-1561.

Field asymmetric wave-form IMS at high electric fields enabled separation of both glyceroand phospholipid isomers.

56. Dwivedi $\mathrm{P}$, Schultz AJ, Jr HHH: Metabolic profiling of human blood by highresolution ion mobility mass spectrometry (IM-MS). Int. J. Mass Spectrom. 2010, 298:7890.

57. Shvartsburg AA, Danielson WF, Smith RD: High-Resolution Differential Ion Mobility Separations Using Helium-Rich Gases. Anal. Chem. 2010, 82:2456-2462.

58. Ray JA, Kushnir MM, Yost RA, Rockwood AL, Wayne Meikle A: Performance enhancement in the measurement of 5 endogenous steroids by LC-MS/MS combined with differential ion mobility spectrometry. Clin. Chim. Acta 2015, 438:330-336.

59. Wojcik R, Webb I, Deng L, Garimella S, Prost S, Ibrahim Y, Baker E, Smith R: Lipid and Glycolipid Isomer Analyses Using Ultra-High Resolution Ion Mobility Spectrometry Separations. Int. J. Mol. Sci. 2017, 18:183.

60. Deng L, Ibrahim YM, Baker ES, Aly NA, Hamid AM, Zhang X, Zheng X, Garimella SVB, Webb IK, Prost SA, et al.: Ion Mobility Separations of Isomers based upon Long Path Length Structures for Lossless Ion Manipulations Combined with Mass Spectrometry. ChemistrySelect 2016, 1:2396-2399.

61. Hankin JA, Murphy RC, Barkley RM, Gijón MA: Ion mobility and tandem mass spectrometry of phosphatidylglycerol and bis(monoacylglycerol) phosphate (BMP). Int. 
J. Mass Spectrom. 2015, 378:255-263.

62. Ahonen L, Fasciotti M, Gennäs GB af, Kotiaho T, Daroda RJ, Eberlin M, Kostiainen R: Separation of steroid isomers by ion mobility mass spectrometry. J. Chromatogr. A 2013, 1310:133-137.

63. Chouinard CD, Cruzeiro VWD, Roitberg AE, Yost RA: Experimental and Theoretical Investigation of Sodiated Multimers of Steroid Epimers with Ion Mobility-Mass Spectrometry. J. Am. Soc. Mass Spectrom. 2017, 28:323-331.

-64. Groessl M, Graf S, Knochenmuss R: High resolution ion mobility-mass spectrometry for separation and identification of isomeric lipids. Analyst 2015, 140:6904-6911.

First documented separation of phospholipid isomers on a high resolution, low field drift tube IM-MS.

65. Šala M, Lísa M, Campbell JL, Holčapek M: Determination of triacylglycerol regioisomers using differential mobility spectrometry. Rapid Commun. Mass Spectrom. 2016, 30:256-264.

66. Maccarone AT, Duldig J, Mitchell TW, Blanksby SJ, Duchoslav E, Campbell JL: Characterization of acyl chain position in unsaturated phosphatidylcholines using differential mobility-mass spectrometry. J. Lipid Res. 2014, 55:1668-1677.

67. Poad BLJ, Green MR, Kirk JM, Tomczyk N, Mitchell TW, Blanksby SJ: High-Pressure Ozone-Induced Dissociation for Lipid Structure Elucidation on Fast Chromatographic Timescales. Anal. Chem. 2017, 89:4223-4229. 